\title{
Quantitative HAADF Imaging of Crystals Containing Heavy Elements: A Comparison with Theory
}

\author{
J. M. LeBeau, ${ }^{*}$ S. D. Findlay, ${ }^{* *}$ X. Wang, ${ }^{* * *}$ A. J. Jacobson, ${ }^{* * *}$ L. J. Allen, ${ }^{* * * *}$ \\ and S. Stemmer* \\ * Materials Department, University of California, Santa Barbara, CA 93106-5050, U.S.A. \\ ** Institute of Engineering Innovation, School of Engineering, The University of Tokyo, \\ Tokyo, 113-8656, Japan \\ *** Department of Chemistry, University of Houston, Houston, TX 77204, U.S.A. \\ **** School of Physics, University of Melbourne, Victoria 3010, Australia
}

Direct comparisons between HAADF simulations and experiments have shown that quantitative agreement can be achieved for single crystalline $\mathrm{SrTiO}_{3}[1,2]$. A previous HAADF comparison study for $\mathrm{PbTiO}_{3}$, with images acquired on an arbitrary scale, exhibited a significant contrast mismatch relative to simulations [3]. To investigate the possibility of an atomic number dependent contrast mismatch, we present a study of single crystalline $\mathrm{PbWO}_{4}$, which contains two cations with large atomic numbers $\left(Z_{\mathrm{Pb}}=82\right.$ and $\left.Z_{\mathrm{W}}=74\right)$. An FEI Titan $80-300 \mathrm{kV} \mathrm{S} / \mathrm{TEM}$ operating at $300 \mathrm{kV}$ was used for HAADF-STEM imaging. The STEM probe forming conditions were set as follows: extraction voltage $4400 \mathrm{kV}$, gun lens 6 , spot size 10 , and a convergence semi-angle of $9.4 \mathrm{mrad}$. The focus was determined using the maximum intensity criterion in both experiments and simulations.

The $\mathrm{PbWO}_{4}$ crystal was observed down the ideal tetragonal scheelite structure [100] zone axis, see inset of Figure 1 for a projected structure. After normalizing experimental image intensities to the incident probe, we present direct comparisons with simulations. Once spatial incoherence is taken into account, near perfect agreement between simulations and experiments is achieved, shown in Figure 1. The effective source size is demonstrated to be independent of atomic number by comparisons with single crystalline $\mathrm{SrTiO}_{3}$.

The importance of incorporating accurate Debye-Waller factors for this study will be emphasized. The variation of the image background intensity will be explored to highlight the importance of image simulations to fully appreciate the subtleties of electron scattering by crystals containing heavy elements. In addition, the stability of the Schottky field emission gun will be discussed in the context of the observed finite source size.

[1] J. M. LeBeau and S. Stemmer, Ultramicroscopy 108, 1653 (2008).

[2] J. M. LeBeau, S. D. Findlay, L. J. Allen, S. Stemmer, Phys. Rev. Lett. 100, 206101 (2008).

[3] D. O. Klenov and S. Stemmer, Ultramicroscopy 106, 889 (2006).

[4] S. S. and J. M. L. acknowledge the NSF for support of this research (grant number DMR0804631). L. J. A. acknowledges support by the Australian Research Council. S. D. F. is supported as a Japan Society for Promotion of Science (JSPS) fellow. 

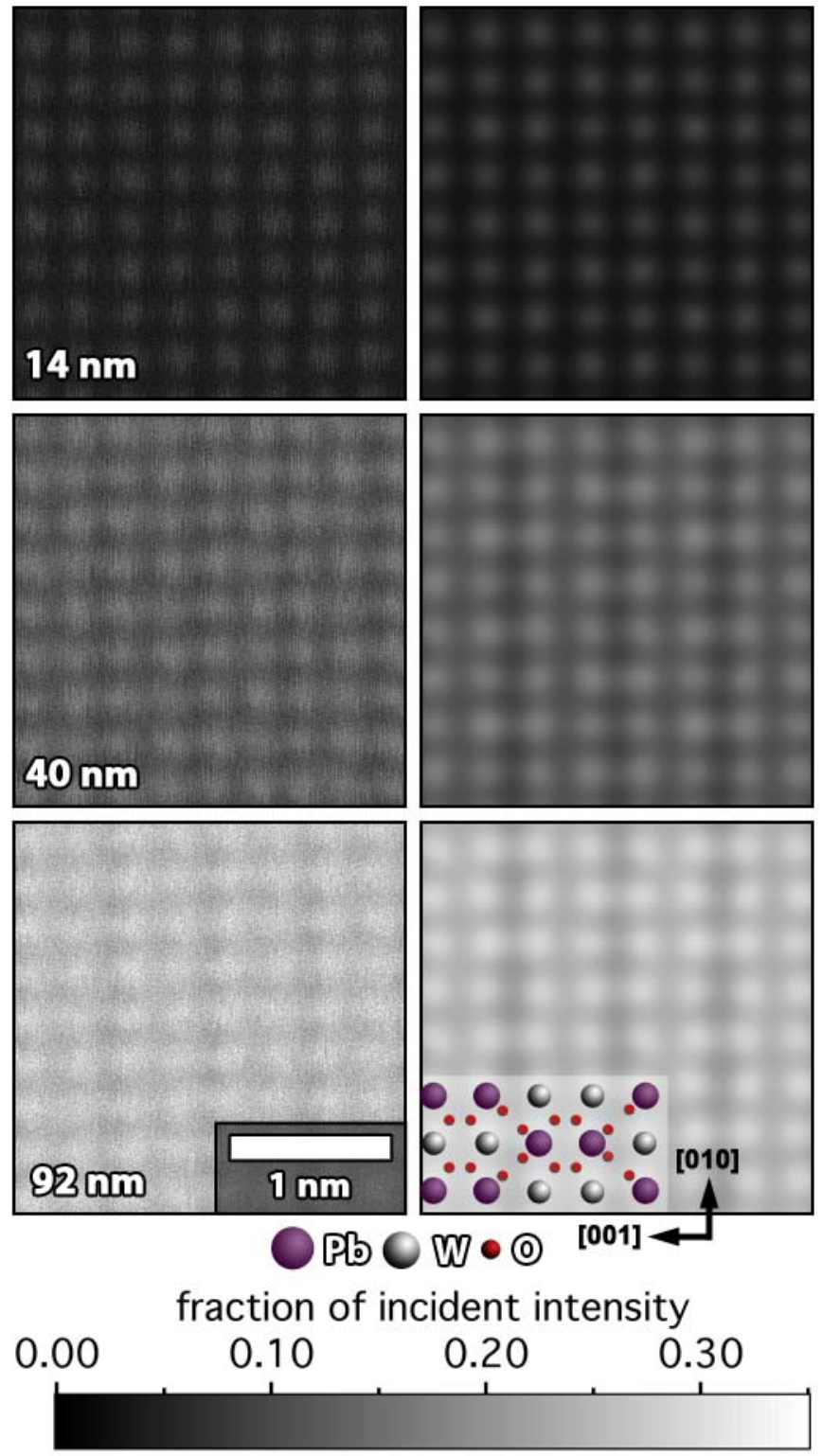

FIG. 1. Experimental (left panels) and simulated (right panels) images for different thicknesses (see labels) of $\mathrm{PbWO}_{4}$. The simulated images have the effects of spatial incoherence taken into account. 